\title{
Bringing Back the Balance: Domain-General Processes Are Also Important in Numerical Cognition
}

\begin{abstract}
Mateusz Hohol ${ }^{1,2 * t}$, Krzysztof Cipora ${ }^{2,3 \dagger}$, Klaus Willmes ${ }^{4}$ and Hans-Christoph Nuerk ${ }^{3,5,6}$
${ }^{1}$ Institute of Philosophy and Sociology of the Polish Academy of Sciences, Warsaw, Poland, ${ }^{2}$ Copernicus Center for Interdisciplinary Studies, Cracow, Poland, ${ }^{3}$ Department of Psychology, University of Tuebingen, Tuebingen, Germany, ${ }^{4}$ Department of Neurology, University Clinic Rheinisch-Westfälische Technische Hochschule Aachen University, Aachen, Germany, ${ }^{5}$ Leibnitz-Institut für Wiessenmedien, Tuebingen, Germany, ${ }^{6}$ LEAD Graduate School and Research Network, Tuebingen, Germany
\end{abstract}

Keywords: numerical cognition, number processing, domain-general processes, cognitive control, inhibition, visual grouping, allocation of attention

In the field of numerical cognition it is often highlighted that the domain-specific systems, referred to as "Approximate Number System" (ANS), or "The Number Sense" (NS) ${ }^{1}$, constitute the basis for mathematical skills (Feigenson et al., 2004; Dehaene, 2011). However, recently, Leibovich et al. (2016) stressed the role of domain-general factors, especially cognitive control, in the formation of elementary numerical abilities. Here we would like to show that these domain-general processes are not only crucial for the formation of number concepts, but in our view, appear to be integral to all aspects of number processing.

Performing both simple numerical processing tasks as well as advanced mathematics requires involvement of domain-general processes apart from domain-specific components. The input material has to be perceived and preprocessed, if necessary stored in (working) memory, processed, and a decision needs to be made. Eventually, all this may lead to a response being given. For the importance of such processes see e.g., LeFevre et al. (2005) and Rousselle et al. (2004). Nevertheless, as numerous studies have shown, domain-general processes cannot be considered only to be providing material to domain-specific processes and then sending back the results. The domain-general processes are rather deeply involved in the information processing path. Focusing on domain-general processes seems necessary from an empirical point of view as well. Over two decades of extensive research aimed to show a relationship between signatures of elementary number/quantity processing ${ }^{2}$ and math skills have not led to consistent results (De Smedt et al., 2013; Lyons et al., 2015). Observed weak relationships between ANS measures and math achievement ( r's $\approx 0.15$ ) often vanish altogether when domain general factors are controlled for (e.g., Gilmore et al., 2013; Szucs et al., 2014). Importantly, not only performance in

\footnotetext{
${ }^{1}$ The term "Approximate Number System" usually refers to the ability of processing nonsymbolic numerosities (e.g., dot patterns; see e.g., Halberda et al., 2008). To the contrary, the term "Number sense" refers to the whole domain of elementary number processing (e.g., Dehaene, 2001), which apart from approximation of nonverbally presented numerosities comprises also subitizing (ability to quickly and effortlessly assess the number of elements of small sets containing less than 4 elements), understanding of the correspondence between symbolic and nonsymbolic numbers, or dot counting. Nevertheless, it should be mentioned, that the definition of the "number sense" differs vastly between authors. Berch (2005) lists 30 components of the number sense proposed by various authors. In general, when used by psychologists, the term "number sense" usually refers to more elementary processing of numerical information (especially nonsymbolic is emphasized), whereas in the field of education it also comprises more complex processes, such as arithmetic knowledge and simple calculation skills.

${ }^{2}$ Behavioral effects observed in tasks requiring number processing such as associating numbers with space, differences in reaction time and/or accuracy of comparing numbers / sets depending on numerical differences between stimuli to be compared.
} 
non-symbolic tasks does not seem to be genuinely related to arithmetic skills. The same holds true also for SpatialNumerical Associations (SNA) and signatures of elementary numerical processing (Cipora and Nuerk, 2013; Cipora et al., 2015, 2016). While any inference from elementary non-symbolic numerical cognition to advanced arithmetic capabilities (the symbol grounding problem; see Leibovich and Ansari, 2016 for review) is far from being clear-cut, there is no doubt that domain-general factors play an important role, both in the case of tasks that are used for measuring NS/ANS (Szucs et al., 2014), and in more complex math performance (Desoete, 2015).

To provide an overview, in the following paragraphs we present three examples that cover domain-general factors operating at varying levels of information processing: from visual grouping through strategic attentional allocation up to inhibition. We discuss how these domain-general processes influence varied areas of numerical cognition, starting from dot set comparisons/estimations through multi-digit number processing up to calculation. These examples are not exhaustive but are aimed at showing the tight relationship between domaingeneral cognitive factors and number processing at different levels.

\section{VISUAL GROUPING}

Despite spatial arrangement of digits being irrelevant to the operation order from a content point of view, Landy and Goldstone (2010) found that it affects performance when solving simple arithmetic problems. For instance, a spatial separation of digits congruent with the rules of arithmetic, such as $2+2 \times 2$ was shown to facilitate calculation performance. RTs are shorter than in incongruent trials, such as $2+2 \times 2$. Thus, the processing of formal operations is systematically biased by the spacing between the symbols. Namely, non-formal properties, such as spatial information, may interfere with knowledge about the order of the operations. This effect does not only hold for arithmetic. Landy and Goldstone (2007) showed that features such as similarity or connectedness affect performance in algebraic problems. Although Landy et al. (2014) interpret these results with respect to the SNA, they may also be understood with reference to general rules of perception, that is, the Gestalt law of proximity (Wertheimer, 1923) and other domain-general factors. Namely, performance in such calculation tasks can be modulated by perceptual factors (influencing visual grouping of the stimuli presented) that affect performance in classical tasks assessing cognitive control (e.g., executive attention tasks). Similar effects were previously demonstrated to influence the interference effect in the flanker task (Eriksen and Eriksen, 1974). Interference is smaller when a target stimulus and distractors ("flankers") are spatially further apart (Miller, 1991).

Summing up, visual grouping processes that are evident in several domain-general cognitive tasks also play a considerable role in number processing at different levels, and to-beinhibited visual stimuli are semantically processed, influencing task performance.

\section{ATTENTION AND CONFLICT MONITORING}

Multi-digit number processing (Hinrichs et al., 1981) also depends largely on domain-general processes (Huber et al., 2016). Multi-digit integers are composed of at least two Arabic digits, arranged in place-value order. Usually processing these numbers requires focusing on digits placed in particular positions, while refraining from processing other numbers. For instance, assessing parity requires focusing only on the unit. On the other hand, comparing magnitudes requires focusing on the highest powers of 10 . Only when digits in the highest power position are equal, one needs to systematically move stepwise toward the lower power positions. Thus, such tasks require an appropriate strategy for the allocation of attentional resources. Unsurprisingly, in both of these tasks, robust compatibility effects were observed (Nuerk et al., 2001, 2015). In the magnitude comparison task RTs are shorter and error rates are lower in case of unit-decade compatible number pairs, i.e., 53_68 $(5<6$ and $3<8)$. In case of incompatible pairs such as $48 \_63(4<6$ but $8>3)$ RTs increase and error rates are higher (Nuerk et al., 2001). The same is true in case of processing magnitudes of decimals, fractions, and negative numbers (Huber et al., 2015). In multidigit number processing tasks, signatures of cognitive control processes known from classical experimental psychology are also observed. For instance, when the proportion of incongruent trials in the Stroop task decreases, interference caused by them increases (MacLeod, 1991). Analogously in the multidigit number comparison task, as the ratio of incompatible vs. compatible trials decreases, the unit-decade compatibility effect gets stronger (Macizo and Herrera, 2013; Huber et al., 2016).

Furthermore, it was demonstrated that participants allocate their attention depending on the task conditions. When the number comparison task comprises only between-decade trials (e.g., 47_62 or 42_57; the decade numbers differ and are decisive for the decision), participants tend to focus on decade magnitude, and the effect of unit-decade compatibility is reduced or disappears totally, because the irrelevant unit causing interference is rarely attended (Huber et al., 2014). When the within-decade filler trials (i.e., trials in which the unit digit is decisive, e.g., 42_47) are introduced, the unitdecade compatibility effect increases, because participants fixate more on the units even in between-decade trials. Eye-fixation behavior and unit interference has been shown to depend on the proportion of fillers: The more often the unit digit is relevant, the larger the proportions of fixation on the unit in the experiment and the larger the unit-decade compatibility effect (Macizo and Herrera, 2011; Moeller et al., 2011; for children see Mann et al., 2011; Huber et al., 2014). Thus, the effects observed in studies on multi-digit number processing (i.e., proportion of congruent and incongruent trials as well as presence of fillers) correspond to the typical pattern of behavior in tasks assessing cognitive control (including executive attention). Both effects could be explained with reference to conflict monitoring, namely, that the participants unconsciously choose a strategy that is optimal for handling given task requirements and the 
overall degree of interference contained in them (Botvinick et al., 2001). On the other hand, when the distance between the unit digits of the two numbers in a number pair is large, unit-decade compatibility appears to be greater (Nuerk et al., 2001). This suggests that this effect is modulated by domainspecific processing of the multi-digit number (Bahnmueller et al., 2015).

Summing up, we can say that domain-general processes involved in allocating attention and conflict monitoring play an essential role in multi-digit number processing as well. Nevertheless, further research is needed to shed more light on the interplay between domain-general and domain-specific factors in this field.

\section{THE ROLE OF INHIBITION}

The previous paragraphs focused on the processing of symbolic numbers. Now effects of domain-general processing on nonsymbolic magnitudes will be discussed. The non-symbolic magnitude comparison task, in which participants compare two numerosities, like sets of dots, is thought to provide a measure of the efficiency of the ANS as assessed by the internal Weber fraction (Piazza et al., 2004, 2010; Gilmore et al., 2011). The ANS is considered to be a domain-specific cognitive module to compare numerosities regardless of the visual properties of objects. In such tasks it is impossible, however, to isolate numerosities from visual properties of the stimuli (Gebuis and Reynvoet, 2012; Szucs et al., 2014). In practice, researchers try to manipulate the visual parameters across trials so that none of these parameters is necessarily linked to the number of dots. The individual trial may be congruent (numerical cue and visual parameters would be leading to the same response) or incongruent (they would lead to opposing responses). This situation leads to a Stroop-like congruency effect, which is expressed as the difference in RT and accuracy between both types of trials (Soltesz et al., 2010). As a rule, children show a larger congruency effect than adults (Szucs et al., 2007). Some explanations take recourse to domain-specific factors, such as an impaired ANS, but others to the influence of cognitive control (Szucs et al., 2013). According to the latter, the congruency effect occurs as a result of distraction by the taskirrelevant visual cue and inefficient inhibition of processing these features in incongruent trials. Thus, the larger congruency effect in children appears as a consequence of their poorer inhibition ability (Huizinga et al., 2006). Furthermore, although performance in typical ANS tasks correlates positively with children's mathematical achievement-which would provide evidence for the importance of the ANS for mathematical skills (Piazza et al., 2010)-this relation is modulated by inhibitory control (Espy et al., 2004). Gilmore et al. (2013) showed that the correlation of performance in ANS tasks and mathematical performance appears to be significant only when incongruent trials (i.e., those requiring inhibition) are considered. Developmental trajectories of refinements of number representations are to some extent congruent with developmental trajectories of improvements in cognitive control (Gilmore et al., 2013).

Summing up, it seems that the role of domain-general processes in a flagship of numerical cognition studies-the non-symbolic comparison task-has been often neglected (see Leibovich et al., 2016, for similar arguments). Executive functions seem to mediate the widely-debated relation between the nonsymbolic comparison task and school math achievement.

\section{CONCLUSIONS}

The above examples were chosen to show that different levels of domain-general processes influence numerical cognition at different levels of numerical processing. Therefore, we argue that their influence should be considered more thoroughly and systematically in the future in both correlation and experimental designs. From the examples we presented, one may get the impression that the simplest aspects of number/quantity processing are influenced by domain-general factors acting relatively late in the sequence of information processing steps, whereas more complex numerical processing is influenced by domain-general factors operating at early stages of information processing. Nevertheless, we would rather refrain from such a conclusion, and instead stress the existence of such influences across all levels both for domain-general factors and elementary number processing.

The aspects we pointed at are only notable examples to illustrate our argument in this article. One could list several other cognitive processes that are tightly related to number processing: Working memory plays a crucial role in performing complex arithmetic operations, where one needs to retrieve facts from long-term memory, conduct several operations, store interim results, and systematically update them to prevent interference (LeFevre et al., 2005). Executive functions play an important role not only in non-symbolic comparisons but correlate strongly with school math performance (Van der Ven et al., 2012). Complex reasoning, mental transformations, and ability to follow appropriate rules play a vital role in conducting mathematical proofs. Linguistic factors were also demonstrated to influence several aspects of numerical processing (see Dowker and Nuerk, 2016 for an overview). We are aware that many other influences exist. However, this opinion article is aimed at pointing out the importance of domain-general influences on different levels of number processing by choosing specific examples. A comprehensive review is beyond the scope of this opinion piece.

Summing up, we strongly emphasize that domain-general factors need to be more widely and thoroughly considered in the field of numerical cognition. In several areas, the role of domaingeneral factors seems to be at least as important (and sometimes apparently more important) than the role of domain-specific factors (Szucs et al., 2014). Therefore, we would like to stress that the crucial role of these processes is by no means limited to NS/ANS and as such, it must be intensely investigated along with domain-specific factors in virtually all fields within numerical cognition. 


\section{AUTHOR CONTRIBUTIONS}

$\mathrm{MH}, \mathrm{KC}, \mathrm{HN}$, and $\mathrm{KW}$ reviewed the literature and developed the theoretical stance. $\mathrm{MH}$ and $\mathrm{KC}$ wrote the manuscript. $\mathrm{MH}$, $\mathrm{KC}, \mathrm{HN}$, and $\mathrm{KW}$ reviewed and accepted its final version. The contribution of $\mathrm{MH}$ and $\mathrm{KC}$ is equal.

\section{FUNDING}

MH is supported by the research grant 2015/19/B/HS1/03310 "Mechanisms of geometric cognition" funded by National

\section{REFERENCES}

Bahnmueller, J., Moeller, K., Mann, A., and Nuerk, H.-C. (2015). On the limits of language influences on numerical cognition - no inversion effects in three-digit number magnitude processing in adults. Front. Psychol. 6, 17-10. doi: 10.3389/fpsyg.2015.01216

Berch, D. B. (2005). Making sense of number sense: implications for children with mathematical disabilities. J. Learn. Disabil. 38, 333-339. doi: 10.1177/00222194050380040901

Botvinick, M. M., Braver, T. S., Barch, D. M., Carter, C. S., and Cohen, J. D. (2001). Conflict monitoring and cognitive control. Psychol. Rev. 108, 624-652. doi: 10.1037/0033-295X.108.3.624

Cipora, K., Hohol, M., Nuerk, H.-C., Willmes, K., Brożek, B., Kucharzyk, B., et al. (2016). Professional mathematicians differ from controls in their spatial-numerical associations. Psychol. Res. 80, 710-726. doi: 10.1007/s00426-015-0677-6

Cipora, K., and Nuerk, H.-C. (2013). Is the SNARC effect related to the level of mathematics? No systematic relationship observed despite more power, more repetitions, and more direct assessment of arithmetic skill. Q. J. Exp. Psychol. 66, 1974-1991. doi: 10.1080/17470218.2013.772215

Cipora, K., Patro, K., and Nuerk, H.-C. (2015). Are spatial-numerical associations a cornerstone for arithmetic learning? The lack of genuine correlations suggests no. Mind Brain Educ. 9, 190-206. doi: 10.1111/mbe.12093

Dehaene, S. (2001). Precis of the number sense. Mind Lang. 16, 16-36. doi: 10.1111/1468-0017.00154

Dehaene, S. (2011). The Number Sense (Revised). Oxford: Oxford University Press.

De Smedt, B., Noël, M.-P., Gilmore, C. K., and Ansari, D. (2013). How do symbolic and non-symbolic numerical magnitude processing skills relate to individual differences in children's mathematical skills? A review of evidence from brain and behavior. Trends Neurosci. Educ. 2, 48-55. Available online at: https://doi. org/10.1016/j.tine.2013.06.001

Desoete, A. (2015). "Cognitive predictors of mathematical abilities and disabilities," in The Oxford Handbook of Numerical Cognition, eds R. Cohen Kadosh and A. Dowker (Oxford: Oxford University Press), 915-932.

Dowker, A., and Nuerk, H.-C. (2016). Linguistic influences on mathematics. Front. Psychol. 7, 1-4. doi: 10.3389/fpsyg.2016.01035

Eriksen, B. A., and Eriksen, C. W. (1974). Effects of noise letters upon the identification of a target letter in a nonsearch task. Percept. Psychophys. 16, 143-149. doi: 10.3758/BF03203267

Espy, K. A., McDiarmid, M. M., Cwik, M. F., Stalets, M. M., Hamby, A., and Senn, T. E. (2004). The contribution of executive functions to emergent mathematic skills in preschool children. Dev. Neuropsychol. 26, 465-486. doi: 10.1207/s15326942dn2601_6

Feigenson, L., Dehaene, S., and Spelke, E. S. (2004). Core systems of number. Trends Cogn. Sci. 8, 307-314. doi: 10.1016/j.tics.2004.05.002

Gebuis, T., and Reynvoet, B. (2012). The interplay between nonsymbolic number and its continuous visual properties. J. Exp. Psychol. Gen. 141, 642-648. doi: $10.1037 / \mathrm{a} 0026218$

Gilmore, C. K., Attridge, N., Clayton, S., Cragg, L., Johnson, S., Marlow, N., et al. (2013). Individual differences in inhibitory control, not non-verbal number acuity, correlate with mathematics achievement. PLoS ONE 8:e67374. doi: 10.1371/journal.pone.0067374
Science Centre, Poland. $\mathrm{HN}$ and $\mathrm{KC}$ are supported by funding of the German Research Foundation (DFG NU 265/3-1) on "Linguistic Influences on Numerical Cognition: A cross-cultural investigation using natural specificities of Polish and German languages."

\section{ACKNOWLEDGMENTS}

The authors would like to thank Julianne Skinner for proofreading.

Gilmore, C. K., Attridge, N., and Inglis, M. (2011). Measuring the approximate number system. Q. J. Exp. Psychol. 64, 2099-2109. doi: 10.1080/17470218.2011.574710

Halberda, J., Mazzocco, M. M., and Feigenson, L. (2008). Individual differences in non-verbal number acuity correlate with maths achievement. Nature 455, 665-668. doi: 10.1038/nature07246

Hinrichs, J. V., Yurko, D. S., and Hu, J.-M. (1981). Two-digit number comparison: use of place information. J. Exp. Psychol. Hum. Percept. Perform. 7, 890-901. doi: 10.1037/0096-1523.7.4.890

Huber, S., Cornelsen, S., Moeller, K., and Nuerk, H.-C. (2015). Toward a model framework of generalized parallel componential processing of multi-symbol numbers. J. Exp. Psychol. Learn. Mem. Cogn. 41, 732-745. doi: $10.1037 / \mathrm{xlm} 0000043$

Huber, S., Mann, A., Nuerk, H.-C., and Moeller, K. (2014). Cognitive control in number magnitude processing: evidence from eye-tracking. Psychol. Res. 78, 539-548. doi: 10.1007/s00426-013-0504-x

Huber, S., Nuerk, H.-C., Willmes, K., and Moeller, K. (2016). A general model framework for multi-symbol number comparison. Psychol. Rev. 123, 667-695. Available online at: https://doi.org/10.1037/rev0000040

Huizinga, M., Dolan, C. V., and van der Molen, M. W. (2006). Age-related change in executive function: developmental trends and a latent variable analysis. Neuropsychologia 44, 2017-2036. doi: 10.1016/j.neuropsychologia.2006.01.010

Landy, D., Allen, C., and Zednik, C. (2014). A perceptual account of symbolic reasoning. Front. Psychol. 5:275. doi: 10.3389/fpsyg.2014.00275

Landy, D., and Goldstone, R. L. (2007). How abstract is symbolic thought? J. Exp. Psychol. Learn. Mem. Cogn. 33, 720-733. doi: 10.1037/0278-7393.3 3.4 .720

Landy, D., and Goldstone, R. L. (2010). Proximity and precedence in arithmetic. Q. J. Exp. Psychol. 63, 1953-1968. doi: 10.1080/174702110037 87619

LeFevre, J.-A., DeStefano, D., Coleman, B., and Shanahan, T. (2005). "Mathematical cognition and working memory," in Handbook of Mathematical Cognition, ed J. I. D. Campbell (New York, NY: Psychology Press), 361-377.

Leibovich, T., and Ansari, D. (2016). The symbol-grounding problem in numerical cognition: a review of theory, evidence, and outstanding questions. Can. J. Exp. Psychol. 70, 12-23. doi: 10.1037/cep0000070

Leibovich, T., Katzin, N., Harel, M., and Henik, A. (2016). From 'sense of number' to 'sense of magnitude' - The role of continuous magnitudes in numerical cognition. Behav. Brain Sci. doi: 10.1017/S0140525X16000960. [Epub ahead of print].

Lyons, I. M., Nuerk, H.-C., and Ansari, D. (2015). Rethinking the implications of numerical ratio effects for understanding the development of representational precision and numerical processing across formats. J. Exp. Psychol. Gen. 144, 1021-1035. doi: 10.1037/xge0000094

Macizo, P., and Herrera, A. (2011). Cognitive control in number processing: evidence from the unit-decade compatibility effect. Acta Psychol. 136, 112-118. doi: 10.1016/j.actpsy.2010.10.008

Macizo, P., and Herrera, A. (2013). The processing of Arabic numbers is under cognitive control. Psychol. Res. 77, 651-658. doi: 10.1007/s00426-012-0456-6

MacLeod, C. M. (1991). Half a century of research on the Stroop effect: an integrative review. Psychol. Bull. 109, 163-203. doi: 10.1037/0033-2909.109.2.163 
Mann, A., Moeller, K., Pixner, S., Kaufmann, L., and Nuerk, H.-C. (2011). Attentional strategies in place-value integration. Zeitschrift für Psychol. 219, 42-49. doi: 10.1027/2151-2604/a000045

Miller, J. (1991). The flanker compatibility effect as a function of visual angle, attentional focus, visual transients, and perceptual load: a search for boundary conditions. Percept. Psychophys. 49, 270-288. doi: 10.3758/BF03214311

Moeller, K., Huber, S., Nuerk, H.-C., and Willmes, K. (2011). Two-digit number processing: holistic, decomposed or hybrid? A computational modelling approach. Psychol. Res. 75, 290-306. doi: 10.1007/s00426-010-0307-2

Nuerk, H.-C., Moeller, K., and Willmes, K. (2015). Multi-digit number processing: overview, conceptual clarifications, and language influences," in The Oxford Handbook of Numerical Cognition, eds R. Cohen Kadosh and A. Dowker (Oxford: Oxford University Press), 106-139.

Nuerk, H.-C., Weger, U., and Willmes, K. (2001). Decade breaks in the mental number line? Putting the tens and units back in different bins. Cognition 82, B25-B33. doi: 10.1016/S0010-0277(01)00142-1

Piazza, M., Facoetti, A., Trussardi, A. N., Berteletti, I., Conte, S., Lucangeli, D., et al. (2010). Developmental trajectory of number acuity reveals a severe impairment in developmental dyscalculia. Cognition 116, 33-41. doi: 10.1016/j.cognition.2010.03.012

Piazza, M., Izard, V., Pinel, P., Le Bihan, D., and Dehaene, S. (2004). Tuning curves for approximate numerosity in the human intraparietal sulcus. Neuron 44, 547-555. doi: 10.1016/j.neuron.2004.10.014

Rousselle, L., Palmers, E., and Noël, M.-P. (2004). Magnitude comparison in preschoolers: what counts? Influence of perceptual variables. J. Exp. Child Psychol. 87, 57-84. doi: 10.1016/j.jecp.2003.10.005

Soltesz, F., Szucs D., and Szucs, L. (2010). Relationships between magnitude representation, counting and memory in 4- to 7-year-old children: a developmental study. Behav. Brain Funct. 6:13. doi: 10.1186/1744-9081-6-13
Szucs, D., Devine, A., Soltesz, F., Nobes, A., and Gabriel, F. (2014). Cognitive components of a mathematical processing network in 9-year-old children. Dev. Sci. 17, 506-524. doi: 10.1111/desc.12144

Szucs, D., Nobes, A., Devine, A., Gabriel, F. C., and Gebuis, T. (2013). Visual stimulus parameters seriously compromise the measurement of approximate number system acuity and comparative effects between adults and children. Front. Psychol. 4, 1-13. doi: 10.3389/fpsyg.2013. 00444

Szucs, D., Soltész, F., Jármi, É., and Csépe, V. (2007). The speed of magnitude processing and executive functions in controlled and automatic number comparison in children: an electro-encephalography study. Behav. Brain Funct. 3, 23-20. doi: 10.1186/1744-9081-3-23

Van der Ven, S. H. G., Kroesbergen, E. H., Boom, J., and Leseman, P. P. M. (2012). The development of executive functions and early mathematics: a dynamic relationship. Br. J. Educ. Psychol. 82, 100-119. doi: 10.1111/j.2044-8279.2011.02035.x

Wertheimer, M. (1923). Untersuchungen zur Lehre von der Gestalt. II. Psychol. Res. 4, 301-350. doi: 10.1007/BF00410640

Conflict of Interest Statement: The authors declare that the research was conducted in the absence of any commercial or financial relationships that could be construed as a potential conflict of interest.

Copyright () 2017 Hohol, Cipora, Willmes and Nuerk. This is an open-access article distributed under the terms of the Creative Commons Attribution License (CC BY). The use, distribution or reproduction in other forums is permitted, provided the original author(s) or licensor are credited and that the original publication in this journal is cited, in accordance with accepted academic practice. No use, distribution or reproduction is permitted which does not comply with these terms. 